\title{
A BOUND ON THE NUMBER OF ENDPOINTS OF THE CUT LOCUS
}

\author{
ROBERT SINCLAIR AND MINORU TANAKA
}

\begin{abstract}
We provide strong experimental evidence for an upper bound on the number of endpoints of the cut locus from a point on a 2-surface of revolution. This bound is equal to the minimal number of intervals of monotone non-increasing or non-decreasing Gaussian curvature along one meridian from one pole to the other.
\end{abstract}

\section{Introduction}

The purpose of this paper is to make use of available software to investigate the nature of an upper bound on the number of endpoints of a pure mathematical object from classical global differential geometry. This paper is therefore intended as an experimental contribution to pure mathematical research, not as a contribution to computational science as such. The main conjecture is presented in Section 2. Chronologically, this conjecture occurred to us before we performed any computational experiments. The remainder of the paper is essentially a series of descriptions of a representative sample of the computational experiments with which we unsuccessfully attempted to falsify our conjecture. The reader can be assured that these experiments have been anything but trivial (the same data set provided a counterexample to another conjecture, as discussed in Section 3.2), and are the result of approximately one year's work. We have used only slightly modified versions of software packages described elsewhere $[\mathbf{2 4}, \mathbf{1 1}, \mathbf{2 5}]$.

We consider only classical analytic two-dimensional Riemannian manifolds (surfaces) of revolution which are embedded in $\mathbb{E}^{3}$. A surface is called a 'classical' surface of revolution if it is obtained by rotating a smooth plane curve in three-dimensional Euclidean space around a straight line lying in its plane and not intersecting it. If the curve is a simple closed smooth curve, then the surface is called a torus of revolution; a simple non-self-intersecting smooth curve is called a cylinder of revolution. We believe that our results also apply to abstract surfaces, which cannot be so embedded, but we have not performed any experiments on such surfaces. An 'abstract' torus of revolution is defined by a two-dimensional Riemannian manifold $S^{1}(a) \times S^{1}(b)$ admitting a Riemannian metric $d t^{2}+m(t)^{2} d \theta^{2}$, where $d t^{2}$ and $d \theta^{2}$ denote the Riemannian metric of the circle of length $a, b$ respectively and $m(t)$ denotes a smooth positive-valued function on the $S^{1}(a)$ of length $a$. An 'abstract' cylinder of revolution is defined by a two-dimensional Riemannian manifold $R^{1} \times S^{1}$ admitting a Riemannian metric $d t^{2}+m(t)^{2} d \theta^{2}$, where $d t^{2}$ and $d \theta^{2}$ denote the Riemannian metric of a real line and of a circle respectively, and $m(t)$ denotes a smooth positive-valued function on $R^{1}$. Note that, by these definitions, all surfaces are abstract.

Shortest paths (geodesics) on surfaces are the paths that frictionless test particles would take if moving freely except for the force required to constrain them to the surface. On a surface of revolution, this constraining force must lie in the plane intersecting both the 
particle itself and the surface's axis of revolution. The torque acting upon the particle therefore has no component in the direction of the surface's axis of symmetry, making the component of the particle's angular momentum in the direction of this axis a conserved quantity. As Clairaut showed in 1733, this allows one to write

$$
\rho \sin \alpha=\text { const }
$$

for all points along a given geodesic, where $\rho$ is the radius of the parallel upon which the point lies, and $\alpha$ the angle that the geodesic makes with a meridian at the same point. In fact, it is well known that the geodesics of a surface of revolution can be found by quadratures; see [27].

The doctoral thesis of Kurt Fleischmann [7] provides an excellent summary of classical results concerning geodesics on surfaces of revolution. He uses the term 'turning circle' to denote a parallel which a geodesic intersects tangentially or approaches asymptotically. In [7, Section 5 of Chapter I], it is stated that there are essentially (excepting some special cases) only three types of geodesics on surfaces of revolution:

- geodesics which oscillate between two parallels - those with two turning circles,

- geodesics which intersect only one parallel tangentially — those with one turning circle, and

- geodesics which intersect every parallel exactly once - those with no turning circle.

Not all surfaces of revolution admit geodesics of all three types. A classification of geodesics on the surface of a circular torus of revolution is given in [5]. The geodesic curves are: (i) the meridian circles, (ii) the two equators, (iii) geodesic curves which wind about the torus crossing the two equators alternately, (iv) geodesic curves which oscillate between two parallels - intersecting these parallels tangentially, and intersecting the outer equator in between - and (v) geodesic curves which intersect the outer equator exactly once, approaching the inner equator asymptotically from above and below. A similar classification is given for the wider class of toroids in [13]. For some illustrations and a more technical discussion, the reader is advised to look at [23, Chapter 7 and Figures 7.1.1 and 7.1.2].

The geometrical object that we wish to study is known as the cut locus. The cut locus from a point $p$ on a Riemannian manifold $M$ is the closure of all points $q$ which admit at least two minimal geodesic segments emanating from $p$. It was first introduced by Poincaré [19], who named it 'ligne de partage', and, independently, by Myers [17, 18]. It depends upon a given surface and a point on that surface, which we will frequently refer to as the starting point. In a rough sense, it is the boundary past which no geodesic from the starting point can be shortest.

On a cylinder of revolution, no geodesic which extends past the meridian opposite the starting point can be shortest, since a shorter path can always be constructed which 'goes the other way around' or makes at least one less full revolution around the surface's axis of revolution. Therefore, cut loci on a cylinder of revolution always include the entire meridian opposite the starting point $[\mathbf{2 8}, \mathbf{3 1}]$.

On the surface of a sphere, geodesics are great circles, which are shortest paths up to, but not beyond, the antipode of the starting point. The cut locus from any point consists therefore of only one point - its antipode.

The cut locus is difficult to determine, due to the fact that it actually consists of two types of points: the first comprising those that are due to two or more distinct equal-length paths meeting at such a point (as in the case of the cut locus from a point on a cylinder of revolution), the other type being conjugate to the starting point, and connected to the starting 
point by only one minimal-length geodesic. These are points along a geodesic which do not move (to first order) under an infinitesimal change in the initial angle of the geodesic at the starting point. One may think of nodes of a vibrating string, or the real optical image of a small object formed by a converging (corresponding to positive Gaussian curvature) lens. Jacobi, in his Lectures on dynamics [12], showed that no geodesic can remain minimizing beyond a conjugate point. It is for this reason that such points must be included in the definition of the cut locus. Only surfaces with regions of positive Gaussian curvature can have conjugate points.

The set of all conjugate points is known as the conjugate locus. Poincaré [19] showed that the endpoints of the cut locus are cusps of the conjugate locus turned towards the starting point. For a surface of revolution, this means that the cut locus will consist of the union of some subarc of the opposite meridian and a finite number of branches, each ending in a conjugate point.

For a formal definition of the conjugate and cut loci, see [22, Chapters II and III]. A detailed discussion of the cut loci of surfaces of revolution appears in [23, Chapter 7]. Conjugate loci need not be closed, nor of finite length [16].

The question of how many endpoints the cut locus may have has already been considered by Myers, who established that the cut locus from a point on a closed analytic surface is a connected linear graph with a finite number of endpoints [17]. We note in passing that one can construct non-analytic surfaces of revolution embedded in $\mathbb{E}^{3}$ with infinitely many endpoints, even ones of strictly positive Gaussian curvature [8].

The conjugate loci from points on paraboloids and hyperboloids were determined in [15], from which one can readily deduce the associated cut loci. These results were extended in [6] to cover the case of what are called 'flattening surfaces'. These are plane-like surfaces of revolution embedded in $\mathbb{E}^{3}$ of the form $z=f\left(\sqrt{x^{2}+y^{2}}\right)$ with monotone non-increasing (and necessarily non-negative) Gaussian curvature from the vertex $(0,0, f(0))$ (the intersection of the surface with its axis of revolution) outwards. The cut loci from points on an even wider class of plane-like surfaces of revolution are given in [29]. These surfaces, called von Mangoldt's surfaces of revolution, satisfy the condition that for any two points $x$ and $y, x$ not further from the vertex than $y$, the Gaussian curvature at $x$ is not less than its value at $y$. For such surfaces and any starting point which is not the vertex, the cut locus is a subset of the meridian opposite the starting point. One may choose to see such results as giving an upper bound on the number of endpoints of the cut locus, as follows: on a plane-like surface of revolution, if the Gaussian curvature is monotone non-decreasing from the vertex outwards, then the number of endpoints of the cut locus of any point is less than two.

Somewhat surprisingly, the cut locus from a point on an abstract surface of revolution need not be connected. An explicit example of a surface and a point from which the cut locus is disconnected is constructed in [30].

The surfaces of revolution of Zoll and Tannery are classical examples of surfaces whose geodesics are all closed [3]. Furthermore, one can compute the cut and conjugate loci exactly in some cases. We will study such an example in Section 3.1.

The cut locus from a general point on a prolate ellipsoid of revolution is a subarc of the opposite meridian. On an oblate ellipsoid of revolution, the cut locus from a general point is a subarc of a parallel; see [25] and [26]. The latter paper contains proofs, which can again be seen as upper bounds on the number of endpoints of the cut locus of a point: if the Gaussian curvature is monotone non-decreasing or non-increasing from a pole to the equator, then the number of endpoints of the cut locus of any point is less than two.

The conjugate loci from points on two classes of tori of revolution embedded in $\mathbb{E}^{3}$ were 
examined in [5] and [13]. A torus of revolution embedded in $\mathbb{E}^{3}$ has cut loci consisting of two intersecting cycles (one the opposite meridian, and the other one a deformed parallel) and, when certain conditions are met, two branches ending in conjugate points. These branches may intersect only one or both of the cycles [10]. Once again, the theorems guarantee an upper bound on the number of endpoints (two) if the Gaussian curvature is monotone non-decreasing or non-increasing from one equator to the other.

Finite upper and lower bounds [4] and estimates [14], all of these in terms of the genus of the surface, for the number of vertices of the cut locus from a point have been given for surfaces without conjugate points (surfaces having non-positive curvature).

In the case of Riemannian manifolds with nonempty boundary and sufficiently small inradius relative to curvature, the cut locus of the boundary is known to be a simple polyhedron with the number of branches bounded by an integer that depends upon the maximum curvature-normalized inradius (see [1, Theorem 1.2] and [2]).

\subsection{Choice of examples}

The types of surfaces that we have studied have clearly been restricted by the software that we have used. In particular, we have only computed cut loci for embeddable surfaces, but we have tried to choose surfaces as different from each other as we could make them.

In this paper, we have not included very many examples for which we computed cut loci but found them to be trivial (having only two endpoints irrespective of the starting point). All of the examples that we have computed, including those not in this paper, support our main conjecture.

The choice of surfaces was therefore neither random nor truly representative (in the space of all surfaces of revolution). Our primary goal was to find a counterexample to our main conjecture, not to observe cut loci 'in general'. We did not attempt to answer the question as to what a 'typical' cut locus might look like, for example. In this context, we believe that our strategy of treating as many different types of surface as we could was appropriate.

\subsection{Visualization}

The style of visualization of surfaces and curves upon them used in this paper has been directly influenced by the school of 'non-photorealistic' rendering, which takes effective communication rather than close resemblance to a photograph as its aim [9]. Our reason for this has been the frequent observation that the hand-drawn illustrations of older geometry textbooks tend not only to be more beautiful than their modern (digitally computed) counterparts, but also to be more truly representative of the geometrical subject matter.

For example, Figure 10 is our attempt to apply the ideas of [21] to clearly represent a surface of revolution using the traditional technique of hatching. (Full-resolution versions of all the figures, in either PNG or PDF format, are available via Appendix A.)

\section{Main conjecture}

A compact Riemannian manifold $(M, g)$ homeomorphic to a 2-sphere is called a 2-sphere of revolution if $M$ admits a point $p$ such that for any two points $q_{1}, q_{2}$ on $M$ with $d\left(p, q_{1}\right)=$ $d\left(p, q_{2}\right)$, where $d($,$) denotes the Riemannian distance function, there exists an isometry$ $f$ on $M$ satisfying $f\left(q_{1}\right)=q_{2}$ and $f(p)=p$. The point $p$ is called a pole of $M$. In [26, Lemma 2.1], it is shown that each pole of $M$ has a unique cut point.

A half-meridian on a 2-sphere of revolution is a minimal geodesic segment joining a pole and its unique cut point. 
CONJECTURE. The number of maximal intervals of non-increasing or non-decreasing Gaussian curvature along a half-meridian of a 2-sphere of revolution, a meridian of a torus of revolution, or a meridian of a cylinder of revolution is an upper bound for the number of endpoints of the cut locus from any point on the surface.

Note that this conjecture is consistent with the main theorems of [10], [26] and [28].

\section{Examples}

We have computed cut loci from many points on each of the surfaces presented in this section. Only three of the surfaces have points from which the cut locus has the same number of endpoints as the number of zones of monotone Gaussian curvature. For the other surfaces, we have found that the number of endpoints is less than our conjectured upper bound.

\subsection{A Zoll surface of revolution}

This is a well-known example from [3, Chapter 4] (especially Sections 4.36 to 4.38 and Figures 4.37 and 4.38), for which the cut locus is known exactly. The example was also treated in [11], where it was used to validate the software described there. We will use the same notation here.

The surface is a Zoll surface of revolution, with (using the same notation as [3])

$$
h(\cos r)=\cos r \frac{\sin ^{2} r}{2},
$$

and an embedding in $\mathbb{E}^{3}$ :

$$
\begin{aligned}
& x(r, \theta)=\sin r \cos \theta \\
& y(r, \theta)=\sin r \sin \theta \\
& z(r, \theta)=\frac{1}{2} \int_{\pi / 2}^{r}(1+\cos u) \sqrt{(1-\cos u)(2-\cos u)\left(2+\cos u+\cos ^{2} u\right)} d u .
\end{aligned}
$$

We compute the cut locus from the point

$$
\left(r_{p}, \theta_{p}\right)=\left(\frac{\pi}{2}, 0\right) .
$$

The cut locus has three endpoints.

The Gaussian curvature is

$$
\frac{8(1+\cos r)\left(1-\cos r+\cos ^{2} r\right)}{\left(2+\cos r-\cos ^{3} r\right)^{3}} .
$$

It is zero at the pole $r=\pi$ (and elsewhere positive), has its global maximum at the other pole $r=0$, a local maximum on one parallel $(r \approx 2.0442)$, and a local minimum on another parallel $(r \approx 1.1154)$. There are no other critical points. The surface therefore has three zones of monotone non-increasing or non-decreasing Gaussian curvature.

This is an example for which the upper bound of our main conjecture is sharp.

\subsection{An example with many endpoints}

Consider the surface given by the parametrization

$$
(\theta, \phi) \mapsto\left(f(\theta) \cos \theta \cos \phi, f(\theta) \cos \theta \sin \phi, \quad \frac{1}{2} f(\theta) \sin \theta\right)
$$

with $\theta \in[-\pi / 2, \pi / 2)$ and $\phi \in[0,2 \pi)$, where 


$$
\begin{aligned}
f(\theta)=2 & -\frac{3}{20} \cos 4 \theta+\frac{1}{1+100 \cos ^{2} \theta} \\
& +\frac{3}{100\left(1+500(\sin \theta-1 / 5)^{2}\right)}+\frac{1}{20\left(1+500 \sin ^{2} \theta\right)},
\end{aligned}
$$

and the starting point is given by

$$
\left(\theta_{0}, \phi_{0}\right)=\left(\pi-\frac{1}{10}, 0\right) .
$$

The surface and cut locus with ten endpoints from this starting point are illustrated in Figure 1. We can be confident that this is indeed the cut locus with the stated number of endpoints by noting that an independent computation of the conjugate locus, presented in Figure 2, shows complete agreement (the endpoints coincide exactly with cusps of the conjugate locus of the appropriate type).

The Gaussian curvature, which is a function of the latitude $\theta$ only, is plotted in Figure 3 . There are clearly twelve intervals (corresponding to zones on the surface) in which it is monotone. For this example, therefore, our conjectured upper bound for the number of endpoints (there are ten endpoints) is twelve.

At one point during our investigations, we thought it plausible that there might be a very small upper bound on the number of endpoints which can appear in any given zone of
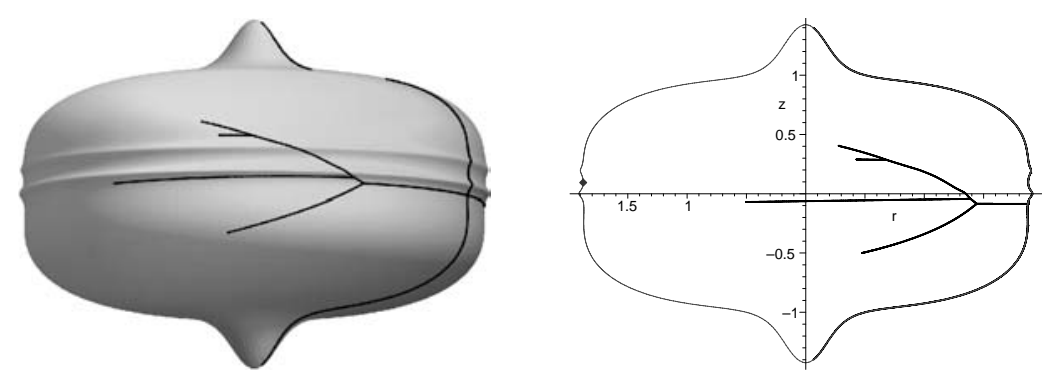

Figure 1: Cut locus with ten endpoints from a point on a surface of revolution given by equation (2). The starting point is at the centre of the dark-grey diamond on the left side of the right-hand (two-dimensional) plot. The coordinates $z$ and $r$ are defined as $[f(\theta) \sin \theta] / 2$ and $f(\theta) \cos (\theta)$ respectively, where $\theta \in[-\pi / 2, \pi / 2)$ and the function $f$ is taken from equation (3).

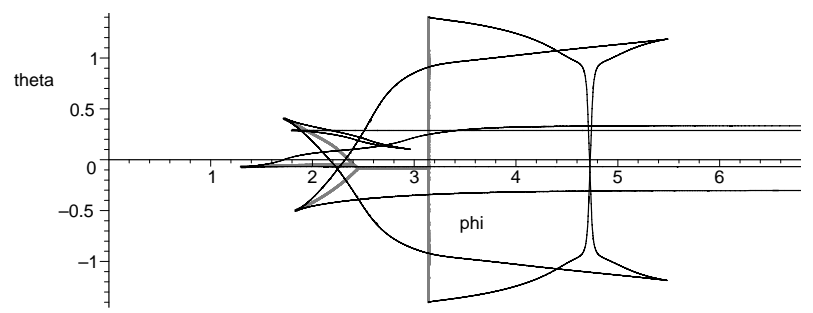

Figure 2: The conjugate locus (thin dark curves) and cut locus (thick grey curves) from a point on the surface of revolution depicted in Figure 1. No point of the cut locus can lie beyond $\phi=\pi$, since this is the meridian opposite the starting point. 
monotone Gaussian curvature. In particular, it seemed possible that this number might be two (perhaps three at end-caps). As Figure 4 shows, however, one can construct examples for which there are zones of monotone Gaussian curvature containing four endpoints. The existence of a counterexample to such a conjecture is, we believe, a sign that our examples have not been poorly chosen.

\subsection{A digression: non-compact surfaces}

The point of this section is to demonstrate that a large number of zones of monotone Gaussian curvature does not necessarily lead to a large number of endpoints of the cut loci from points. Non-compact surfaces with an infinite number of such zones are ideal examples for this purpose:

$$
(u, v) \mapsto\left(\frac{2-\sin 10 v}{1+v^{2}} \cos u, \frac{2-\sin 10 v}{1+v^{2}} \sin u, v\right) .
$$

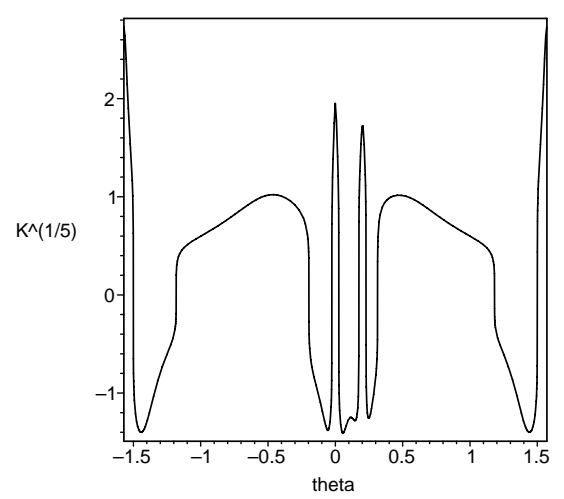

Figure 3: The Gaussian curvature $K$ (what has been plotted is actually, $K^{1 / 5}$, or $-|K|^{1 / 5}$ if $K$ is negative) as a function of latitude $\theta$ on the surface of revolution given by equation (2). The main point is that one can identify twelve zones in which the Gaussian curvature is monotone increasing or decreasing.

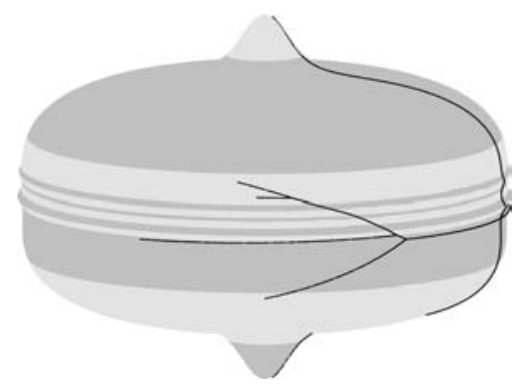

Figure 4: The cut locus from a point on the surface of revolution given by equation (2). The twelve zones in which the Gaussian curvature is monotone increasing or decreasing are depicted using grey tones. One can see that there is one zone containing four endpoints. This is a counterexample to a conjecture which would have claimed there to be less than four in any zone. 
What we find is that there are only four endpoints from the starting point $(2,0,0)$, although there are apparently infinitely many cusps of the conjugate locus (only some of which are apparent in Figures 5 and 6; one can find more by following geodesics as they wind ever further around the axis of symmetry).
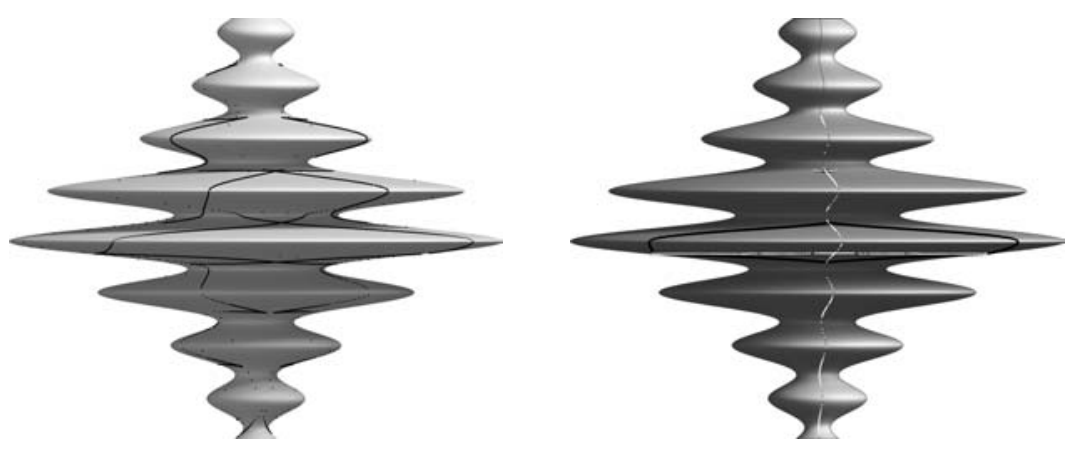

Figure 5: Left: Conjugate points. Right: Cut points (white) and conjugate points with $|v| \leqslant \pi$ (black). The surface is defined by equation (4). The starting point is $(2,0,0)$.

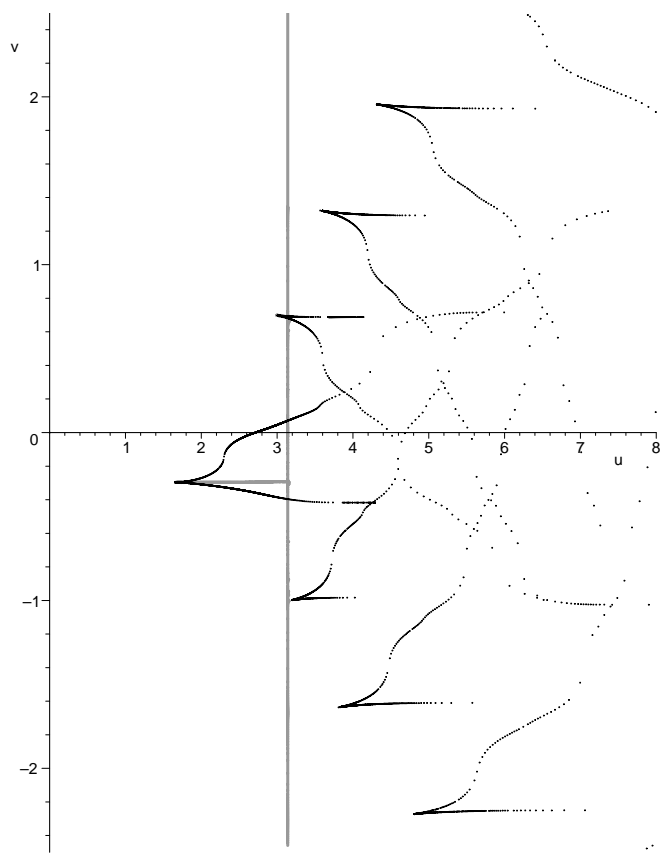

Figure 6: Cut (grey) and conjugate (black) points. The cylinder of revolution is defined by equation (4). The starting point is $(2,0,0)$. The horizontal coordinate is the angle around the axis of symmetry. The cut locus includes the opposite meridian to the starting point (the vertical line at $u=\pi$ ), but cusps of the conjugate locus which lie beyond this cannot be associated with endpoints of the cut locus. 


\subsubsection{A 'periodic'surface}

\section{Consider}

$$
(u, v) \mapsto([2+\sin v] \cos u,[2+\sin v] \sin u, v)
$$

with the three starting points

$$
(2,0,0), \quad\left(3,0, \frac{\pi}{2}\right) \text { and }\left(1,0, \frac{-\pi}{2}\right) .
$$

The fact, illustrated in Figure 7 , that the cut locus from the point $(2,0,0)$ is the union of the opposite meridian (to the starting point) with a subarc of the parallel $v=\pi$ follows from the proof of [10, Proposition 3.2]. Similarly, the cut locus from the point $(3,0, \pi / 2)$ is the union of the opposite meridian with a subarc of the parallel $v=\pi / 2$.

In the case of the starting point $(1,0,-\pi / 2)$, the parallel $v=-\pi / 2$ is itself one geodesic. All other geodesic curves passing through this starting point must also pass through every parallel, since the starting point lies on a parallel of globally minimum radius. It follows immediately that no geodesic curve emanating from this starting point has a conjugate point, and, therefore, that the cut locus consists of only the meridian opposite the starting point.

\subsection{A deformed sphere}

We consider

$$
(\theta, \phi) \mapsto(f(\theta) \cdot \cos \theta \cdot \cos \phi, f(\theta) \cdot \cos \theta \cdot \sin \phi, f(\theta) \cdot \sin \theta),
$$

where

$$
f(\theta)=2+\frac{1}{20} \cos (4 \theta)+\frac{1}{20} \sin (3 \theta) .
$$

There are four zones of monotone Gaussian curvature. We have computed cut loci from the starting points $\left(\theta_{0}, 0\right)$, where $\theta_{0}$ was $-1.3,-1,-0.6,-0.3,0,0.3,0.6,1$ and 1.3 . The maximum number of endpoints observed was four; see Figure 8.
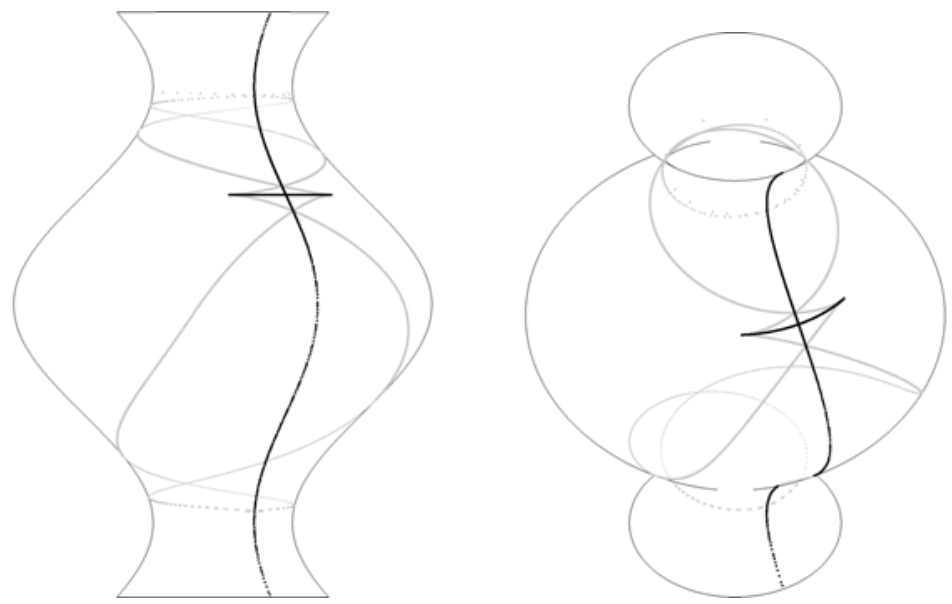

Figure 7: Two views of the cut locus (dark) and conjugate locus (grey) from a point on the periodic surface of revolution given by equation (5). The cut locus consists of one entire meridian (that is, of infinite length) and exactly one other segment. The conjugate locus does not extend beyond the two necks depicted. 


\subsection{A mushroom}

Here, we consider

$$
(\theta, \phi) \mapsto\left(f(\theta) \cdot \cos \theta \cdot \cos \phi, f(\theta) \cdot \cos \theta \cdot \sin \phi, \frac{1}{2} f(\theta) \cdot \sin \theta\right),
$$

where

$$
f(\theta)=2+\frac{1}{2} \cos (4 \theta)+\frac{1}{2} \sin (3 \theta) .
$$

There are five zones of monotone Gaussian curvature. In Figure (9), the starting point is at $\left(\theta_{0}, 0\right)$, where $\theta_{0}$ is $-1.3,-1,-0.6,-0.3,0,0.3,0.6,1$ and 1.3 respectively, from left to right and top to bottom. The maximum observed number of endpoints was four (see Figure 10).

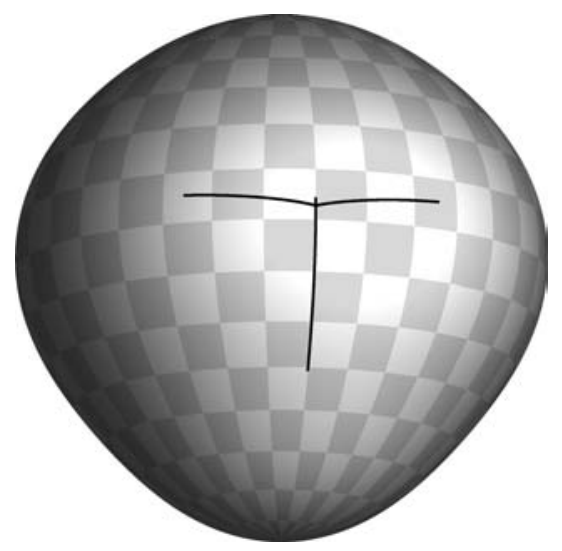

Figure 8: The cut locus from the point $(41 / 20,0,0)$ on the deformed sphere defined by equation (6).
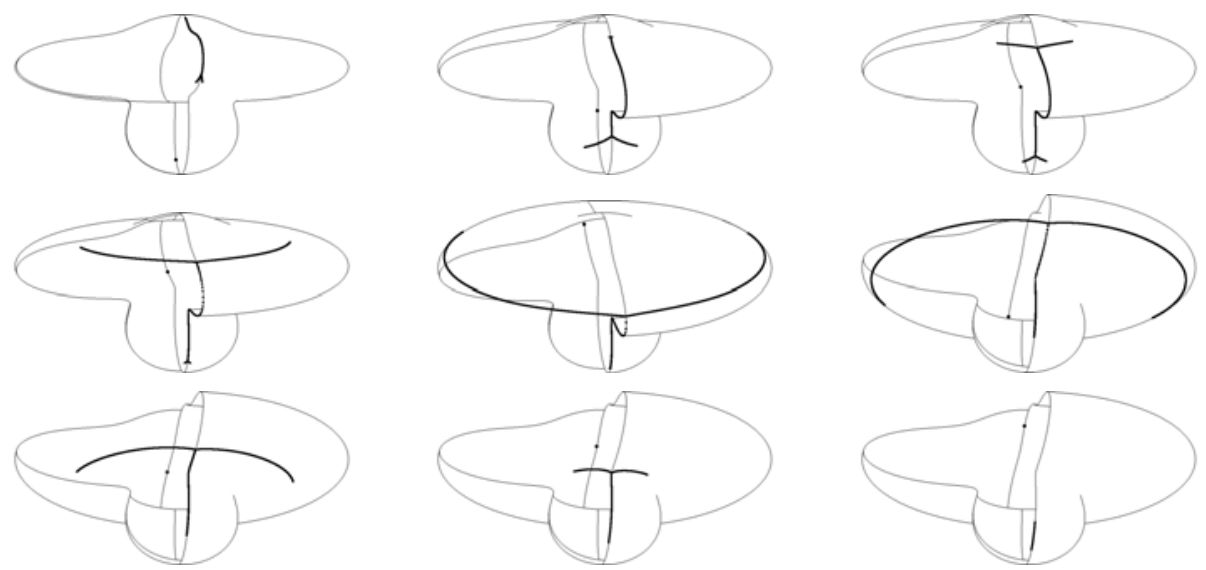

Figure 9: Computer-generated sketches of cut loci from starting points on the mushroom surface defined by equation (7) at $\theta_{0}=-1.3,-1,-0.6,-0.3,0,0.3,0.6,1,1.3$, from top-left to bottom-right respectively. 


\subsection{Convex surface with cut locus of many endpoints}

Here we add a controlled number of ripples (parallels) to a sphere:

$$
(\theta, \phi) \mapsto(f(\theta) \cos \theta \cos \phi, f(\theta) \cos \theta \sin \phi, f(\theta) \sin \theta)
$$

with

$$
f(\theta)=2+\frac{1}{60} \sin 9 \theta .
$$

There are nine zones of monotone Gaussian curvature.

This example is one of a family of deformed spheres. It would seem that one can increase the number of endpoints by simply changing the function $f$ (the number of endpoints seems to be given by the coefficient of $\theta$ ).

We have computed an approximation to the cut locus using the software tool Thaw [11]. The actual starting-point coordinates were $(1.999992,-0.000210,-0.000087)$. The number of subdivisions (this controls the number of triangles) was $N=6$. Since one of the endpoints was still unclear, we also computed the conjugate locus. The two computational methods agree where Thaw's output is clear (see Figure 11), and the cusps of the conjugate locus make it possible to ascertain the number of endpoints. We find nine endpoints.

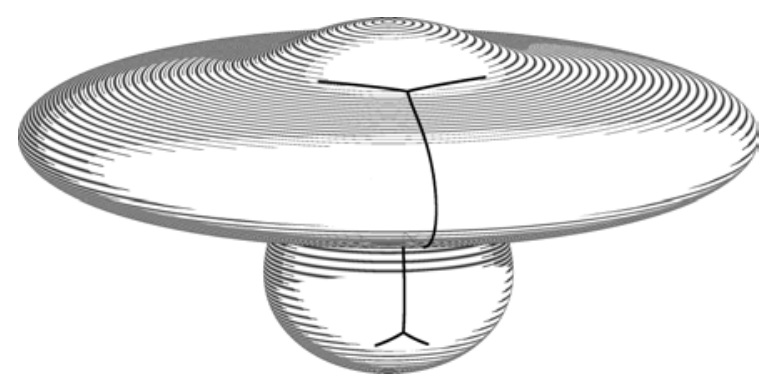

Figure 10: The cut locus from a point on the mushroom surface defined by equation (7).

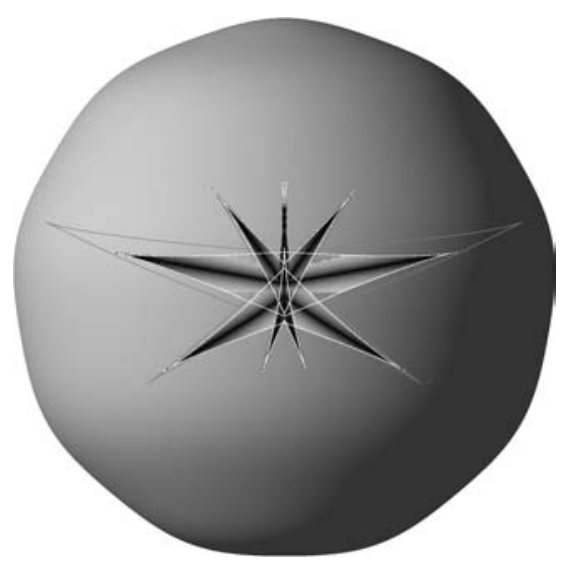

Figure 11: The cut locus and conjugate locus (white where less than $\pi$ from the starting point, grey where further) of the deformed spherical surface given by equation (8). 


\subsection{A non-standard torus of revolution}

We consider

$$
\begin{aligned}
(u, v) \mapsto( & \left\{\frac{1}{6}[\sin (3 u)+5] \cos (u)+2\right\} \sin v \\
& \left.\left\{\frac{1}{6}[\sin (3 u)+5] \cos (u)+2\right\} \cos v, \frac{1}{6}[\sin (3 u)+5] \sin u\right) .
\end{aligned}
$$

This surface has ten zones of monotone (as a function of the parameter $u$, with $v$ fixed) Gaussian curvature.

The cut locus is forked (see Figure 12; compare it with Figure 15), and has four endpoints.

\section{The role of monotonicity of the Gaussian curvature}

Here we present two experimental studies of surfaces of revolution, which we deform to destroy the monotonicity of the Gaussian curvature. In both cases, it appears that the appearance of new endpoints of the cut loci coincides exactly with the loss of monotonicity. Both examples demonstrate that our main conjecture is 'sharp' with respect to at least some deformations of some surfaces.

\subsection{Forking of the cut locus of a deformed ellipsoid}

Consider the following family of surfaces of revolution:

$$
(\theta, \phi) \mapsto\left(f(\theta) \cos \theta \cos \phi, f(\theta) \cos \theta \sin \phi, \frac{1}{2} f(\theta) \sin \theta\right)
$$

where

$$
f(\theta)=2-\omega \cos 4 \theta,
$$

and $\omega=0$ corresponds to the ellipsoid of revolution $x^{2} / 4+y^{2} / 4+z^{2}=1$.
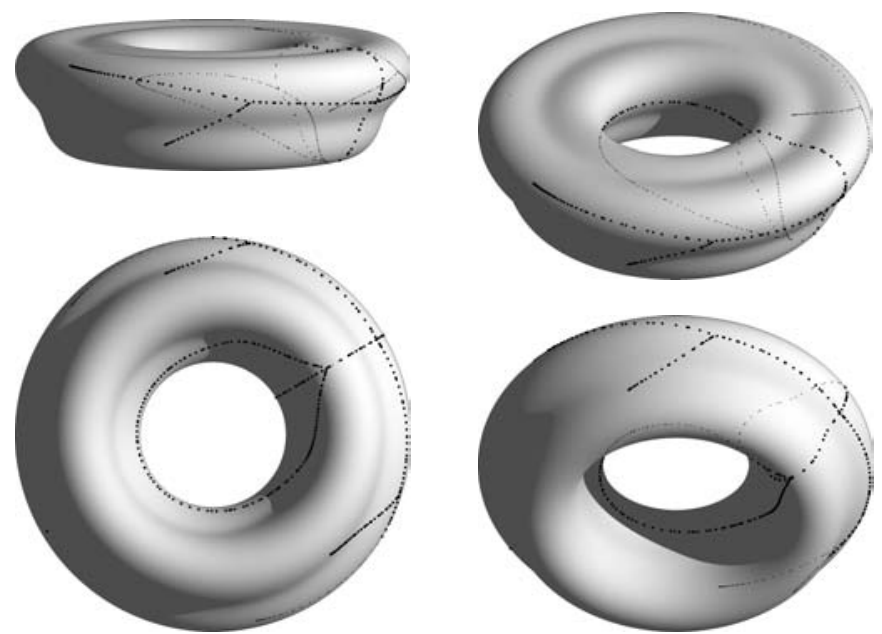

Figure 12: Cut locus on the surface of revolution (torus) given by equation (9) from the point $(0,17 / 6,0)$. 
The Gaussian curvature is monotone non-decreasing on a meridian from pole to equator if $\omega$ is less than

$$
\begin{aligned}
\omega_{c}= & \frac{1066}{11301} \\
+\sqrt[3]{5(6140855+135612 \sqrt{3190})} & \left(\frac{-16}{56505}+\sqrt[3]{5(6140855+135612 \sqrt{3190})}\right. \\
& \left.\times\left(\frac{192}{6498778225} \sqrt{3190}-\frac{19650736}{14688538544145}\right)\right)
\end{aligned}
$$

$\approx 0.033841079$

Thus, [26, Lemma 4.1] (which states that the cut locus of a point on the equator of a surface, for which the Gaussian curvature is monotone non-decreasing on a meridian from a pole to any point on the equator, is a subset of the equator) applies when $\omega<\omega_{c}$, and the cut locus of a point on the equator will be a subset of the equator in that case. Is this condition sharp? In other words, does $\omega>\omega_{c}$ (lack of monotonicity) imply that the cut locus will not be a subset of the equator?

We can investigate this question numerically, by computing the cut locus (using a modified version of Loki [24]) for various values of $\omega$. To start with, we have the two extreme cases illustrated in Figure 13.

It is clear from the figure that there are values of $\omega>\omega_{c}$ for which the cut locus from a point on the equator is not a subset of the equator. We will now determine an approximation to the value $\omega_{n}$, at which the forking of the cut locus begins. If it appears that $\omega_{n}=\omega_{c}$, then we will conclude that the monotonicity condition in [26, Lemma 4.1] is sharp, at least for this family of surfaces.

The illustrations of Figure 14 are of projections of the cut locus onto a plane tangential to the surface at the point in the cut locus where the two branches originate. Angles are represented faithfully. Computing close to $\omega_{c}$ requires very small error tolerances, and therefore quite intensive calculations. In the illustrations for $\omega=0.034$ and $\omega=0.033$, the grey dots are the result of a computation to the same error tolerance as for $\omega=0.036$ and $\omega=0.035$, and the black dots come from computations with an error tolerance one tenth of that.
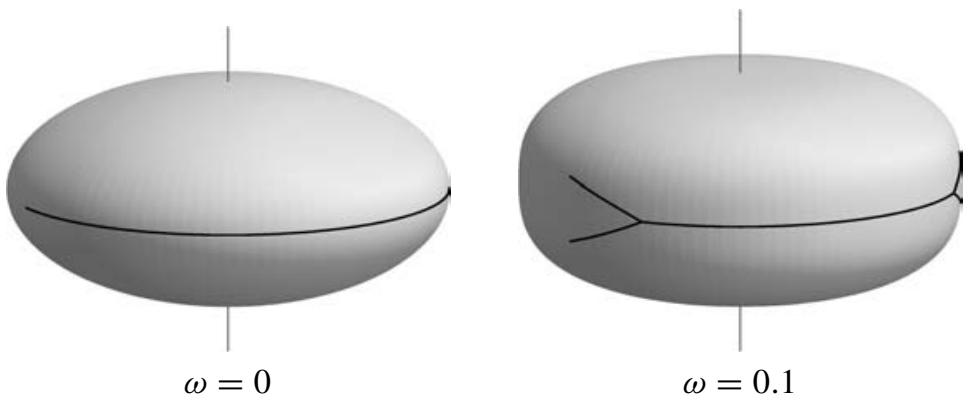

Figure 13: Cut loci from points on the equator of the one-parameter family of deformed ellipsoids given by equation (10) for different values of this parameter $\omega$. One can see that the number of endpoints has changed as $\omega$ increased from zero. 
Table 1: Size of the forks at the endpoints of the cut loci of various surfaces of revolution given by equation (10).

\begin{tabular}{ccc}
\hline$\omega$ & $d\left(p_{Y}, p_{0}\right)-d\left(p_{e}, p_{0}\right)$ & $\sqrt{d\left(p_{Y}, p_{0}\right)-d\left(p_{e}, p_{0}\right)}$ \\
\hline 0.034 & 0.0000200 & 0.00447 \\
0.035 & 0.0009144 & 0.03024 \\
0.036 & 0.0030911 & 0.05560 \\
\hline
\end{tabular}

These more intensive computations (which took several weeks) have been restricted to what is necessary to identify the endpoints and the positions where the branches of the cut locus meet the equator. In the final illustration $(\omega=0.033), \omega<\omega_{c}$, so [26, Lemma 4.1] applies, and the cut locus must be a subset of the equator. In this plot, the vertical axes have been stretched to indicate the extremely small deviations of the numerical results from the equator.

We can tabulate the difference between the distance from $p_{0}$ to one of the endpoints $p_{e}$, and the distance from $p_{0}$ to the point of intersection of the two branches $p_{Y}$ as a function of $\omega$; see Table 1 .
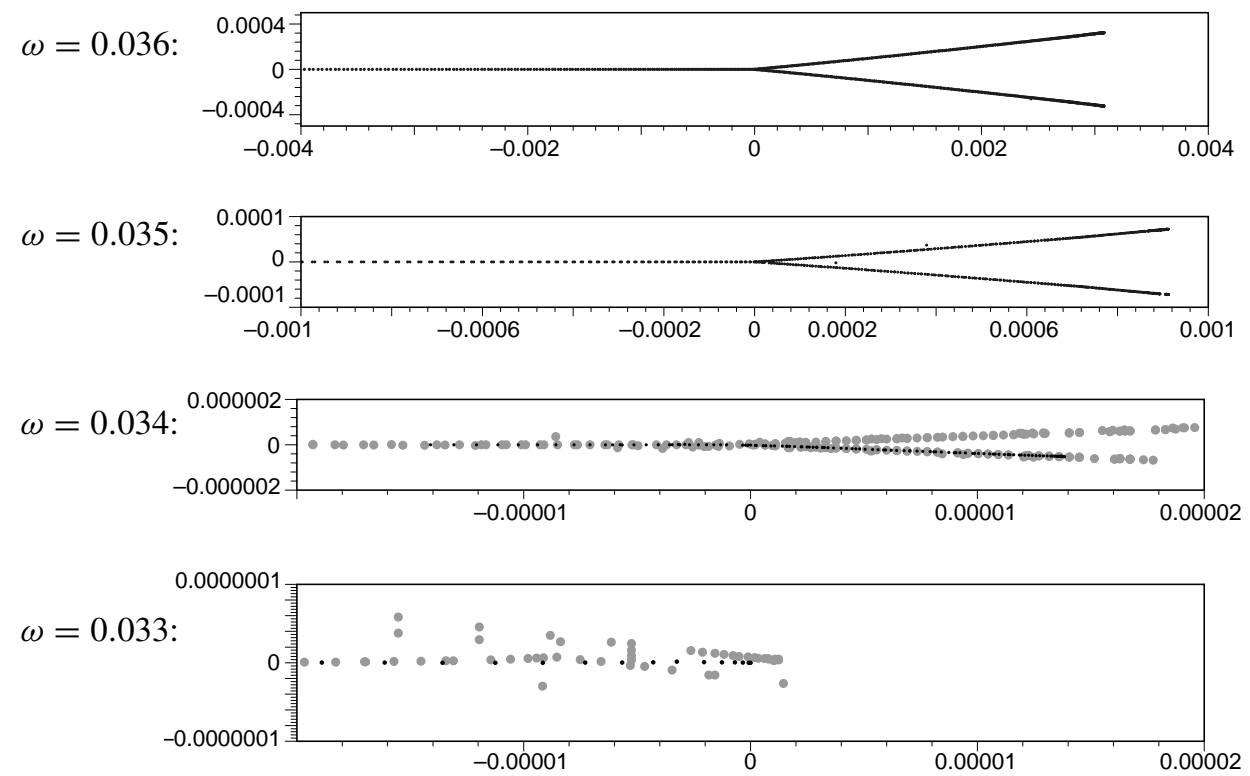

Figure 14: Forking behaviour in the cut locus from points on the equator of the family of deformed ellipsoids given by equation (10) for different values of the parameter $\omega$. The critical value of $\omega$ is approximately 0.03384 . 
We observe that there is an $\omega_{n}$ such that

$$
\sqrt{d\left(p_{Y}\left(p_{0}, \omega\right), p_{0}\right)-d\left(p_{e}\left(p_{0}, \omega\right), p_{0}\right)} \propto\left(\omega-\omega_{n}\right)
$$

for $0<\omega-\omega_{n} \ll 1$, and we can use this proportionality to estimate $\omega_{n}$, the value of $\omega$ at which the forking behaviour begins (numerically). We find that

$$
\omega_{n} \approx 0.03383
$$

which is extremely close to $\omega_{c} \approx 0.03384$.

This numerical experiment therefore strongly suggests that the monotonicity condition in [26, Lemma 4.1] and presumably also in the main conjecture of this paper, is sharp, at least with respect to some deformations of some surfaces.

\subsection{Forking of the cut locus of a torus embedded in $\mathbb{E}^{3}$}

Consider the family of tori

$$
(u, v) \mapsto((2+\cos u) \cos v,(2+\cos u) \sin v, \alpha \sin u)
$$

for positive real values of $\alpha$.

If the Gaussian curvature is monotone non-decreasing along a meridian from the inner to the outer equator, then the cut locus from a point on the outer equator will have two endpoints [10]. Using the properties of Jacobian elliptic functions, it is straightforward to show that this monotonicity condition is satisfied for

$$
\alpha \leqslant \alpha_{c}=\frac{\sqrt{30}}{5} \approx 1.09545 .
$$

For $\alpha>\alpha_{c}$, one notices in experiments that the number of endpoints doubles to four. This phenomenon was first observed many years ago (but not published) by Prof. S. Markvorsen and J. Gravesen of the Technical University of Denmark, using software developed by J. Rebel [20]. One of the motivations for developing Loki [24] was to provide independent confirmation, and also to obtain higher-resolution images, of this process. Two examples, computed here using Loki, for $\alpha=0.7$ and 1.5 respectively, are presented in Figure 15.
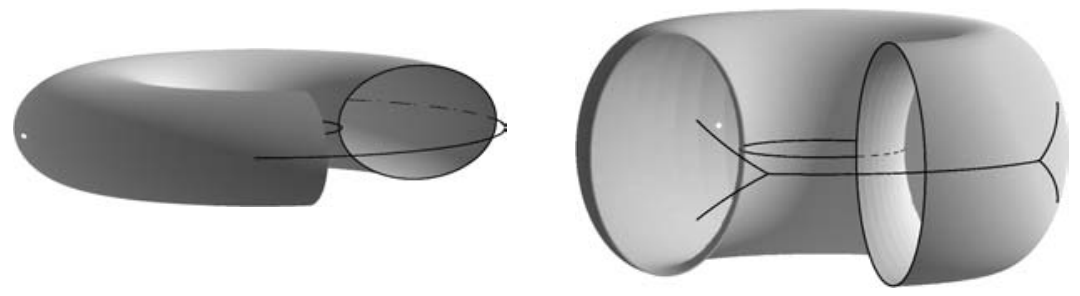

Figure 15: Tori given by $(u, v) \mapsto((2+\cos u) \cos v,(2+\cos u) \sin v, \alpha \sin u)$ with $\alpha=0.7$ (left) and $\alpha=1.5$ (right). The cut locus from a point (shown in white) on the outer equator is drawn in black in each case. For $\alpha=0.7$, there are two endpoints, for $\alpha=1.5$ there are four. 
Table 2: Size of the forks at the endpoints of the cut loci of various tori of revolution with elliptic generating curves.

\begin{tabular}{l|llcc}
\hline$\alpha$ & $d\left(p, p_{Y}\right)$ & $d\left(p, p_{\min }\right)$ & $d\left(p, p_{Y}\right)-d\left(p, p_{\min }\right)$ & $\sqrt{d\left(p, p_{Y}\right)-d\left(p, p_{\min }\right)}$ \\
\hline 1.12 & 6.0868 & 6.074885 & 0.0119 & 0.109 \\
1.11 & 6.03695 & 6.032720 & 0.00423 & 0.0650 \\
1.1 & 5.98523 & 5.984787 & 0.00044 & 0.021 \\
1.098 & 5.974556 & 5.9744161 & 0.000140 & 0.0118 \\
\hline
\end{tabular}

Further experimentation with Loki for values of $\alpha$ closer to unity suggests that the forking disappears in a continuous manner as $\alpha$ approaches $\alpha_{c}$ from above. Figure 16 illustrates this, with four cut loci overlaid on the right-hand side of the figure for the values $\alpha=1.12$, $\alpha=1.11, \alpha=1.1$ and $\alpha=1.098$. The left-hand side of Figure 16 still clearly shows the forking at $\alpha=1.098$, although the size is extremely small for this value of $\alpha$.

More detailed examination shows that there is an approximate linear relationship (asymptotically, as $\alpha$ approaches $\alpha_{c}$ ) between $\alpha$ and the size of the fork of the form

$$
\alpha-\alpha_{c} \propto \sqrt{d\left(p, p_{Y}\right)-d\left(p, p_{\min }\right)} .
$$

From Table 2 we can conclude that

$$
\alpha_{c} \approx 1.0953
$$

which is indeed close to $\sqrt{30} / 5$.
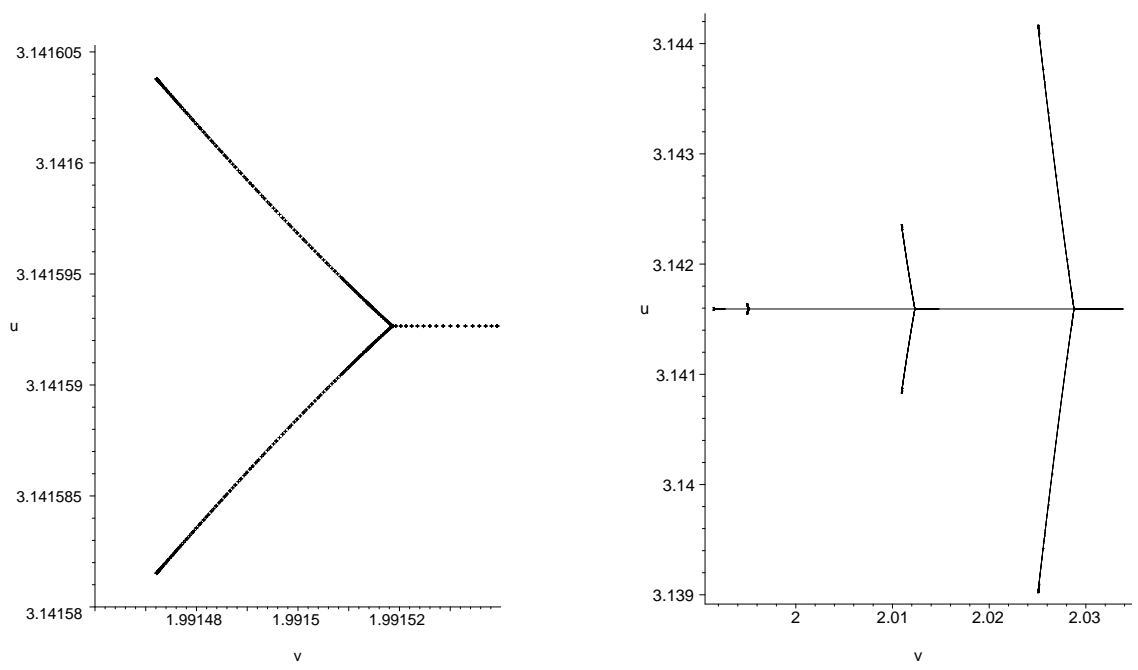

Figure 16: The vanishing of the forking at the endpoints of the cut locus of a torus of revolution with elliptic generating curve as the eccentricity vanishes (from right to left). The right-hand illustration is an overlay of four different sets of data. The left-hand illustration shows a magnified view of the smallest of these. 


\section{Conclusion}

We have provided substantial numerical evidence for an upper bound on the number of endpoints of cut loci from points on surfaces of revolution, this bound being the minimal number of zones of monotone non-increasing or non-decreasing Gaussian curvature. Such a bound follows naturally from earlier work on surfaces of revolution $[29,26,10]$ which established, in various different contexts, that the number of endpoints is less than or equal to two if the number of zones of monotone non-increasing or non-decreasing Gaussian curvature is two. We have also demonstrated that a non-trivial lower bound is unlikely to exist. It is our hope that these computational experiments will one day lead to theorems.

\section{Appendix A. Figures}

Full-resolution versions of all the figures, in either PNG or PDF format, can be found at

$$
\text { http://www.lms.ac.uk/jcm/9/lms2005-018/appendix-a. }
$$

Acknowledgements. The authors would like to thank Prof. S. Markvorsen and J. Gravesen of the Technical University of Denmark for sharing their computational results and checking our analysis of the phenomenon of forking of the cut locus on tori of revolution with elliptic generating curves.

\section{References}

1. Stephanie B. Alexander and Richard L. Bishop, 'Spines and homology of thin Riemannian manifolds with boundary', Adv. Math. 155 (2000) 23-48. 2000] 24

2. Stephanie B. Alexander and Richard L. Bishop, 'Spines and topology of thin Riemannian manifolds', Trans. Amer. Math. Soc. 355 (2003) 4933-4954. 24

3. Arthur L. Besse, 'Manifolds all of whose geodesics are closed', Ergeb. Math. Grenzgeb. 93 (Springer, Berlin, 1978). 23, 25

4. David B. BleEcKer, 'Cut loci of closed surfaces without conjugate points', Colloq. Math. 44 (1981) 263-276. 24

5. Gilbert A. Bliss, 'The geodesic lines on the anchor ring', Ann. of Math. (2) 4 (1902) $1-21.22,24$

6. Doug Elerath, 'An improved Toponogov comparison theorem for nonnegatively curved manifolds', J. Differential Geom. 15 (1980) 187-216. 23

7. K. FleischmanN, 'Die geodätischen Linien auf Rotationsflächen', Inaugural Dissertation, Seyffarth, Liegnitz, 1915, http://www-gdz.sub.uni-goettingen.de/cgi-bin/digbib.cgi?PPN317487884. 22

8. Herman Gluck and David A. Singer, 'Scattering of geodesic fields, II', Ann. of Math. 110 (1979) 205-225. 23

9. Bruce Gooch and Amy Gooch, Non-photorealistic rendering (A. K. Peters, Natick, MA, 2001). 24

10. Jens Gravesen, Steen Markvorsen, Robert Sinclair and Minoru Tanaka, 'The cut locus of a torus of revolution', Asian J. Math. 9 (2005) 103-120.24, 25, 29, 35, 37 
11. Jin-ICHi Itoh and Robert Sinclair, 'Thaw: a tool for approximating cut loci on a triangulation of a surface', Experiment. Math. 13 (2004) 309-325. 21, 25, 31

12. Carl Gustav Jаков JACOBI, 'Vorlesungen über Dynamik', Gesammelte Werke, C. G. J. Jacobi, 2nd edn, supplementary volume (ed. A. Clebsch and E. Lottner; Georg Reimer, Berlin, 1884). 23

13. B. F. Kimball, 'Geodesics on a toroid', Amer. J. Math. 52 (1930) 29-52. 22, 24

14. Chuan Yu Ma, 'The number of vertices of cut loci $C(A)$ of closed Riemannian surfaces', Nanjing Daxue Xuebao Shuxue Bannian Kan 4 (1987) 106-108. 24

15. Hans von MANGOLdT, 'Ueber die jenigen Punkte auf positiv gekrümmten Flächen, welche die Eigenschaft haben, dass die von ihnen ausgehenden geodätischen Linien nie aufhören, kürzeste Linien zu sein', J. Reine Angew. Math. 91 (1881) 23-53. 23

16. Christophe M. Margerin, 'General conjugate loci are not closed', Differential geometry: Riemannian geometry, ed. Robert Greene and S.T. Yau, Proc. Symposia in Pure Math. 54, Part 3 (Amer. Math. Soc., Providence, RI, 1993) 465-478. 23

17. Sumner Byron Myers, 'Connections between differential geometry and topology: I. Simply connected surfaces', Duke Math. J. 1 (1935) 376-391. 22, 23

18. Sumner Byron Myers, 'Connections between differential geometry and topology: II. Closed surfaces', Duke Math. J. 2 (1936) 95-102. 22

19. Henri Poincaré, 'Sur les Lignes géodésiques des surfaces convexes', Trans. Amer. Math. Soc. 6 (1905) 237-274. 22, 23

20. Johannes Rebel, 'Tower of Babylon', MSc Thesis, Eksamensprojekt nr. 1995-07, Mathematical Institute, Technical University of Denmark, 1995. 35

21. Takafumi Saito and Tokitchiro Takahashi, 'Comprehensible rendering of 3-D shapes', Computer Graphics 24, Number 4 (1990) 197-206. 24

22. TAKashi SaKai, Riemannian geometry, Transl. Math. Monogr. 149 (Amer. Math. Soc., Providence, RI, 1996). 23

23. Katsuhiro Shiohama, TAKashi Shioya and Minoru TANaKa, The geometry of total curvature on complete open surfaces, Cambridge Tracts in Math. 159 (Cambridge University Press, 2003). 22, 23

24. Robert Sinclair and Minoru Tanaka, 'Loki: software for computing cut loci', Experiment. Math. 11 (2002) 1-25. 21, 33, 35

25. Robert Sinclair, 'On the last geometric statement of Jacobi', Experiment. Math. 12 (2003) 477-485. 21, 23

26. Robert Sinclair and Minoru Tanaka, 'The cut locus of a 2-sphere of revolution and Toponogov's comparison theorem', preprint,

http://homepage.mac.com/r_m_sinclair/Cutlocus2sphere_draft.pdf. $23,24,25,33,34,35,37$

27. Dirk J. Struik, Lectures on classical differential geometry, 2nd edn (Dover Publications, New York, 1988). 22

28. Kazuhiro Tamura, On the cut locus of a complete Riemannian manifold homeomorphic to a cylinder, MSc Thesis, Graduate Course of Science, Tokai University, Japan, 2004. 22, 25

29. Minoru TANaKa, 'On the cut loci of a von Mangoldt's surface of revolution', J. Math. Soc. Japan 44 (1992) 631-641. 23, 37 
30. Minoru TANAKA, 'On a characterization of a surface of revolution with many poles', Mem. Fac. Sci., Kyushu Univ. Ser. A, Mathematics 46 (1992) 251-268. 23

31. Yuichi Tsuji, 'On a cut locus of a complete Riemannian manifold homeomorphic to cylinder', Proc. School of Sci., Tokai Univ. 32 (1997) 23-34. 22

Robert Sinclair sinclair@math.u-ryukyu.ac.jp

http://homepage.mac.com/r_m_sinclair/

Department of Mathematical Sciences

Faculty of Science

University of the Ryukyus

Nishihara City

Okinawa 903-0213

Japan

Minoru Tanaka m-tanaka@sm.u-tokai.ac.jp

Department of Mathematics

Tokai University

Hiratsuka City

Kanagawa 259-1292

Japan 